\title{
QUANTUM COMPUTING FOR DESIGNING BEHAVIORAL MODEL AND QUANTUM MACHINE LEARNING ON A HUMANOID ROBOT
}

\author{
Jacob Viertel \& Mohamed Aburaia
}
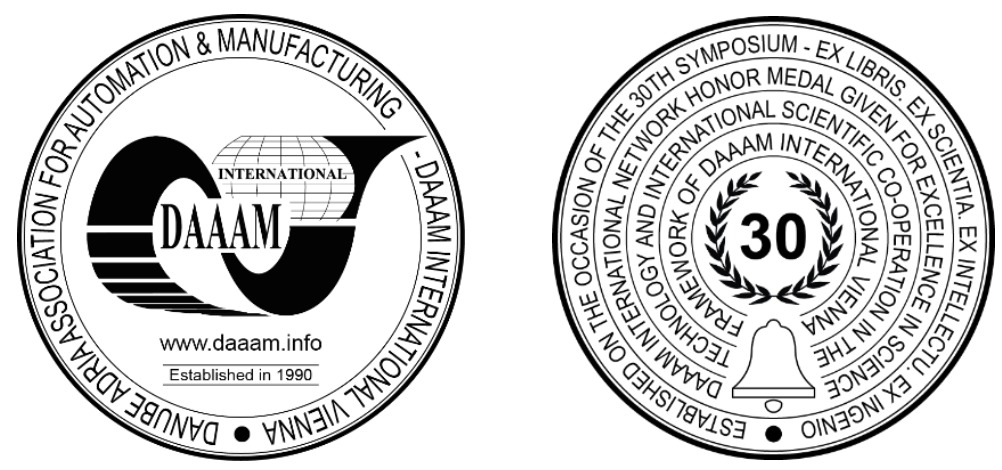

This Publication has to be referred as: Viertel, J[acob] \& Aburaia, M[ohamed] (2021). Quantum Computing for Designing Behavioural Model and Quantum Machine Learning on a Humanoid Robot, Proceedings of the 32nd DAAAM International Symposium, pp.0599-0606, B. Katalinic (Ed.), Published by DAAAM International, ISBN 978-3-90273433-4, ISSN 1726-9679, Vienna, Austria

DOI: $10.2507 / 32$ nd.daaam.proceedings.085

\begin{abstract}
Today's robots are controlled with classical computers that make decisions based on algorithms designed for a classical computing architecture. However, the amount of data increases continuously - a fact which pushes classical computers that control these robots to their limits. In comparison to classical computing, fundamentally different concepts of physics are the basis of quantum computing and thereby quantum computing has the potential to solve specific computational problems more efficiently. In addition to the aforementioned complications, the subgroup of robots that are subject to the research of this paper, namely humanoid robots, not only need more computational capacity but also require an algorithmic design based on a behavioral model which avoids causing antipathy when interacting with human beings. Using a real quantum computer with quantum phenomena such as superposition, entanglement, and quantum parallelism, new behavioral models and algorithms can be designed to improve human robot interaction and speed up machine learning processes. This paper promotes a hybrid-controlled robot using the combination of a classical computer- and quantum computer. With four quantum circuits to define a behavioral model and three different quantum machine learning algorithms we show the advantage of the hybrid-controlled robot.
\end{abstract}

Keywords: Quantum Robotics; Human-Robot-Interaction; Quantum-Machine-Learning; Grover-Algorithm, QuantumSupport-Vector-Machine.

\section{Introduction}

Classical computers can perfectly optimize smaller systems, but when it comes to larger systems such as transportation routes or product pricing, they only achieve incremental improvements [1]. With increasing problem size, the amount of data often grows exponentially. This faster than polynomial growth of data leads to an accumulation of computational costs that in most cases can be hard to handle by a classical computer. Not only the safety standards have to increase when robots collaborate with human beings, but also the quality of the interaction between them. Humanoid robots need to express resemblances of feelings and emotions like aggression or fear in order to increase the level of communication and create a higher acceptance of a robot participating in a conversation with humans [2]. The Uncanny-Valley theory [3] relates the human affinity to the human-like appearance and motions of a robot. 
If the robots' appearance is somewhere in-between the humanlike-robot and the cartoon-like-robot its appearance will arouse human antipathy. Designing a behavioral model with classical computing is cost-intensive and further stresses the capacity of classical computers. With the deterministic nature of classical computing, it is difficult to imitate the nondeterministic behavioral model of a human being. Therefore, within this paper we use the probabilistic nature of quantum computing to create a complex behavioral model with lower computational costs than a classical behavioral model. Areas like quantum machine learning [4], [5] or mood and emotion generation with quantum computing [6], [7] have been researched more frequently in recent publications to solve problems in a way that is impossible for classical computers. One contribution of this paper is an approach to use an adaptation of the Grover algorithm in order to solve several variations of the Shortest Path problem. The Grover algorithm adaptation can analyse a graph if a path between starting node and target node exists. Despite the early state of quantum computers, the Grover adaption can keep up with various classical algorithms such as Dijkstra or Bellman Ford.

The goal of this paper is not to outperform known classical computing systems, but simply to show the raw potential of quantum humanoid robots. It shows that it is possible to overcome the problem of setting up a control system for a humanoid robot that consists of two different computers that are based on fundamentally different concepts of physics. Additionally, this paper introduces various methodologies how to transfer a classical robotic related problem in order to use quantum computing to solve them. With respect to the diversity of the research field quantum robotics, this paper focuses on motion generation with quantum computing and solving robotic related problems such as the Shortest Path problem or vision. According to the concepts developed in this paper quantum robots have classical sensors, a hybridcomputer, and classical effectors. The sensor data from the classical computer must be converted with respect to the different physics of the quantum computer before being further processed within a quantum circuit. Afterwards, the results of these quantum circuits had to be mapped again to trigger any actions of the classical effectors.

\section{Fundamentals of Quantum Circuits}

Every operation or gate in quantum computing can be represented as a matrix transformation of a state vector along a sphere. A quantum bit or qubit is a basic unit to store information in quantum computing and it represents the most trivial two-state quantum system [8]. The transformations in quantum computing happen in Hilbert Space, which is a specific type of vector-space. Regarding a quantum behavioral model, within the Hilbert Space the probabilistic nature of measuring a quantum circuit can be used to design cost-efficient characteristic quantum circuits that imitate different behaviours similar to the Braitenberg Vehicle concept [9]. The main idea of the Braitenberg Vehicle is to mimic complex behaviour like fear or shyness with simple methods. As the research area of quantum cognition is built on the theory that the human behaviour model is closer related to quantum computing than to classical computing [10], quantum computing is more suitable to define the artificial behavioral model of a robot that interacts with human beings.

Furthermore, quantum computing can also speed up algorithmic problems using quantum algorithms such as Shor [12] or Grover [11]. The Grover algorithm [11] is a quantum algorithm that is used for searching unstructured data and it provides a quadratic speed-up in comparison to the best classical computing algorithm. The heart of the Grover algorithm is the so-called Grover-oracle. Adapting this oracle to solve a problem that is applicable to robotics is the main challenge during the implementation of the Grover algorithm. Another potential for the use of quantum machine learning is due to the fact that quantum computing is superior in comparison to classical computing in terms of calculating the dot-product. The dot-product is used when calculating the inner product. Therefore, quantum computing has greater potential doing the Swap-Test [13] to classify data points in comparison to classical computing.

This paper proposes a hybrid-controlled robot using a classical computer for the main control system that can outsource specific tasks to a quantum computer. This setup causes an additional problem that is not of quantum nature only such as handling the noise. A collaborative problem of classical and quantum computing is that most data are stored on classical computers, therefore, they must be mapped suitable for quantum computers onto qubits before algorithms like Quantum Support-Vector-Machine can use them for classification.

\section{Quantum Motions Generators}

A quantum motion generator circuit uses quantum phenomena like entanglement and superposition to generate motions with behaviours such as:

- Deterministic

- Classical probabilistic

- Quantum probabilistic

- Mixed probabilistic

The motion behaviours are encoded onto the control register as follows:

- $|00\rangle$ Deterministic

- $\quad 10\rangle$ Quantum Probabilistic

- $\quad 01\rangle$ Quantum Probabilistic

- $\quad 11\rangle$ Classical Probabilistic 
In the four behaviour models above the operator $U$ in the circuit below is defined as unitary matrix and has no effect during the calculation. Fig.[1] shows the circuit to realize all four behavioral motions.

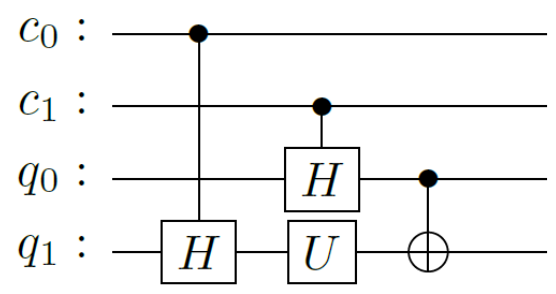

Fig. 1. The circuit can be divided in two quantum registers, target- and control-register. According to $c_{0}$ and $c_{1}$ the three behaviours deterministic, classical, and quantum will be executed.

Editing the transformation matrix $\mathrm{U}$, a mixed probabilistic behaviour can be generated, which is a mixture of both classical and quantum probabilistic. Eq. [1] shows the matrix of the single transformation operator $\mathrm{U}$.

$U=\left[\begin{array}{cc}\cos \frac{\theta}{2} & -e^{-i \omega} \sin \frac{\theta}{2} \\ e^{i \omega} \sin \frac{\theta}{2} & e^{i \lambda+i \omega} \cos \frac{\theta}{2}\end{array}\right]$

The circuit was applied to the greeting behaviour of the biped robot. With the deterministic or probabilistic behaviour, the robots greeting behaviour were not suitable for a high-quality interaction with human beings. The deterministic behaviour is too monotone and the probabilistic behaviour too random to be considered human-like. With quantum probabilistic or mixed probabilistic behaviour model, the robots greeting became more human-like.

\section{Quantum Behavioral Modelling}

To define a more complex behavioral model of the robot, two additional quantum circuits are needed. With the mood and handed circuit it is possible to create a behavioral model of the humanoid robot with less computational cost than other models including convolutional nets such as proposed in [14]. The mood-circuit influences the speed and range of the motion execution according to its internal state by mapping two moods, happy and exhausted, onto the circumference of a qubit. For the classical computer to adjust speed and range of the robot the mapped state vector from the quantum behavioral model must be measured. Fig. [2] shows the circuit to obtain the state vectors orientation using phases kickback within an inverse Quantum Fourier Transform.

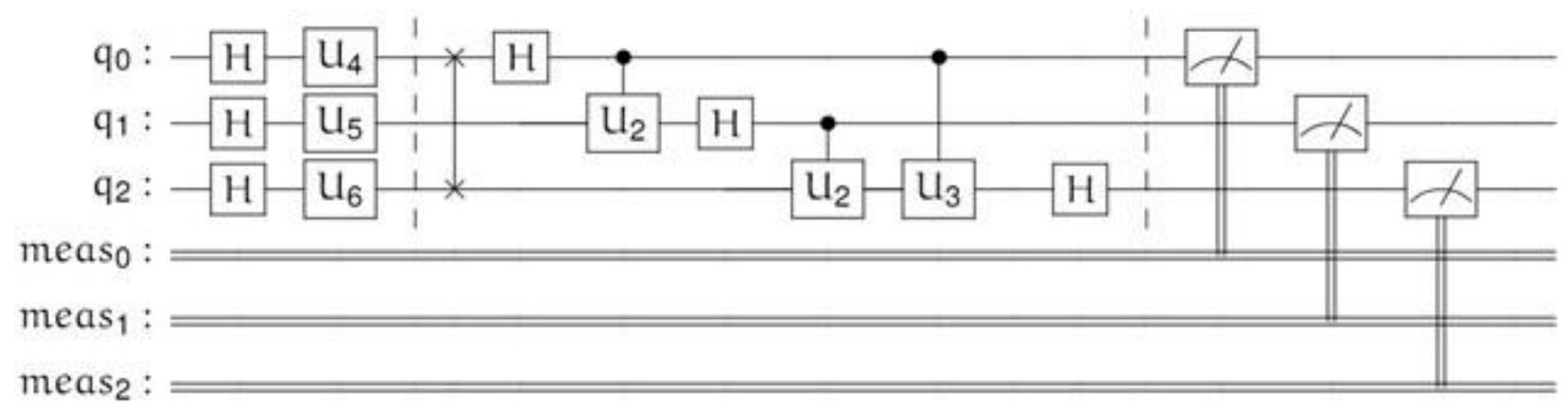

Fig. 2. The illustrated circuit obtains the quantum state vectors orientation in order to be further processed on the classical computer.

Even before a human is born, he/she/it already has the predisposition to be either left- or right-handed [15]. To add such a native characteristic to the behavioral model of the robot in order to form a more in-depth persona with little computational cost, the handed circuit defines the robots' strong hand. To define the robot's handedness, the quantum circuit empathizes left- or right-handed motions with probabilistic distribution. Fig. [3] shows the circuit for a left-handed persona, that is divided into three parts with dashed lines.

The Grover circuits for left- and right-handed characteristics are both initialized with two Hadamard gates at the beginning of the circuit. The second part of the circuit, the so-called Grover oracle, is the only part that varies for the leftand right-handed persona. The last part of the circuit, the so-called reflection around average is the same for both circuits. 


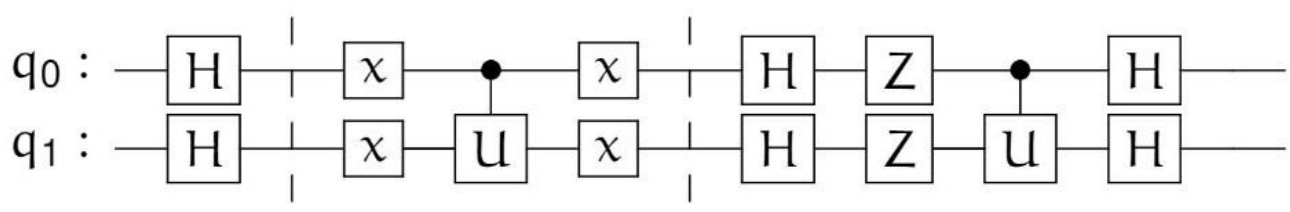

Fig. 3. The circuit for the right-handed persona varies only in the middle part of the circuit.

The last circuit for the quantum behavioral modelling realizes the first quantum random walk [16] on a humanoid biped. This circuit is used to train the robot a method to find its target using randomized patterns. The robot tries to find a person in a room by randomly changing its direction and generating a random walking or turning behaviour. When the robot finds the target person, it will approach the person and greet him/her with a quantum generated waving motion. This is the first realization of the quantum random walk on a humanoid biped robot.

\section{Quantum Machine Learning}

This approach uses the famous Grover Oracle to solve the algorithmic problem of the Shortest Path. To adapt the Grover oracle to the Shortest Path problem, the edges of a graph are encoded as qubits and the nodes as total symmetric Boolean functions. Fig. [4] shows the example graph with its six vertices and seven edges.

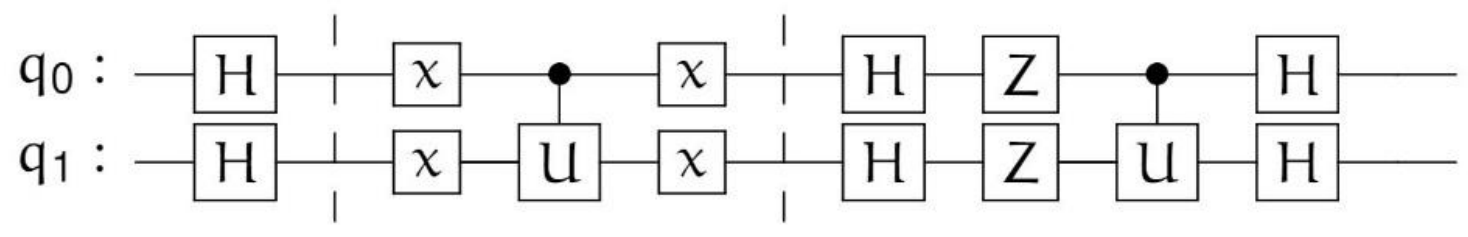

Fig. 4. A is the starting node and $\mathrm{F}$ is the target node.

With this approach it is possible to find a valid path in a given graph in $O\left(\frac{\pi}{4} \sqrt{\frac{2^{E}}{M}}\right)$ with $\mathrm{M}$ being the valid solutions, $\mathrm{V}$ being the numbers of vertices and $\mathrm{E}$ being the number of edges. More nodes or vertices are nowadays not possible, as the number of qubits is limited on real quantum computers and on quantum simulations. The runtime $\mathrm{O}$ of three classical algorithms and our Grover adaption can be seen below with $\mathrm{V}$ representing the number of vertices and $\mathrm{E}$ representing the number of edges:

1. Bellman Ford $O(V * E)=42$

2. Dijkstra $O((V+E) \log (V))=13$

3. Floyd-Warshall $O\left(V^{3}\right)=216$

4. Grover oracle $O\left(\frac{\pi}{4} \sqrt{\left(\frac{2^{E}}{M}\right)}+\frac{\pi}{4} \sqrt{\left(\frac{2^{E}}{M-1}\right)}+\frac{\pi}{4} \sqrt{\left(\frac{2^{E}}{M-2}\right)} \approx 20\right.$

In addition to the Shortest Path problem the Grover adaption can also solve problems such as:

- Does a path exist?

- What is a path?

- How many edges does the path have?

After designing the Grover oracle, the problem Does a path exist? can be solved using the quantum counting algorithm [18]. In this algorithm, the Grover oracle is used in combination with an inverse Quantum Fourier Transform. The result of this quantum circuit is needed in order to distinguish how often the Grover circuit has to be iterated to obtain a valid solution. In the runtime equation for the Grover oracle, $O\left(\frac{\pi}{4} \sqrt{\frac{2^{E}}{M}}\right)$, the number of solutions is represented with $M$. Using a wrong number of iterations will lead to a decreased accuracy.

After obtaining the number of valid paths the other three problems can be solved:

- What is a valid path?

- How many edges does the path have?

- What is the shortest valid path?

For the example graph, the result of the quantum counting algorithm indicated $M=3$. Fig. [5] shows the probabilities of measuring one of the three possible paths for the example graph. 


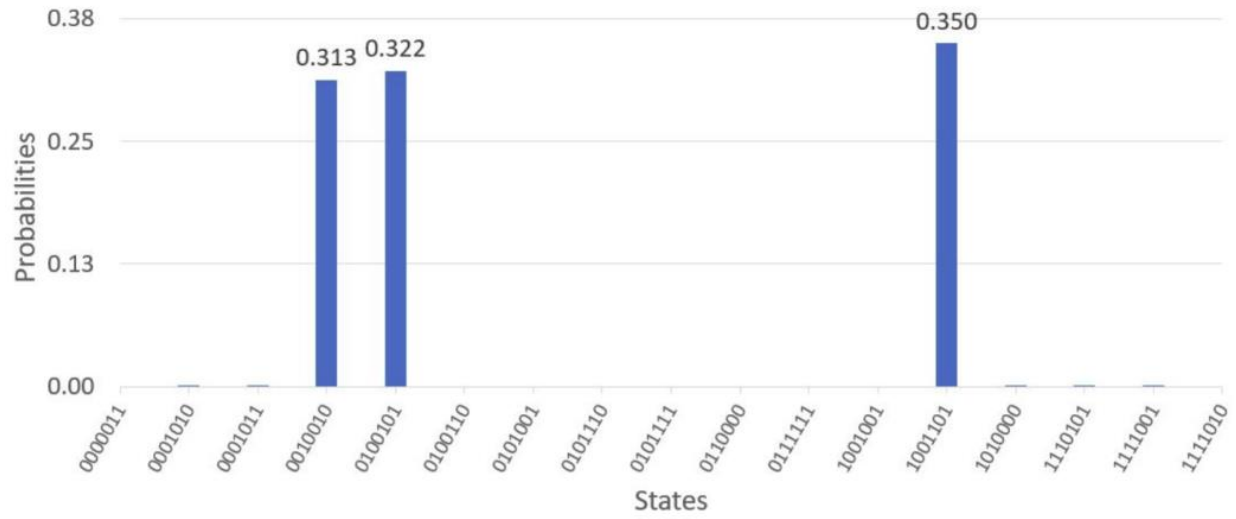

Fig. 5. When a measurement is done after three iterations, one of the three paths is measured. The paths active edges are encoded in the qubit string at the $\mathrm{X}$-axis.

The methodology of finding the Shortest Path requires, additional to the classical Grover oracle two components:

- A Quantum-Adder

- A Quantum-Comparator

After finding a valid path, the costs of the path are calculated using a quantum circuit of half- and full adder. If this path was the first one that was found, it will define the new threshold. Afterwards the Grover oracle is edited in order to no longer consider the founded path as a valid solution. In the next phase, the costs of the next founded path will be compared with this threshold using the quantum comparator circuit. Fig. [6] shows the entire circuit for solving the Shortest Path problem.

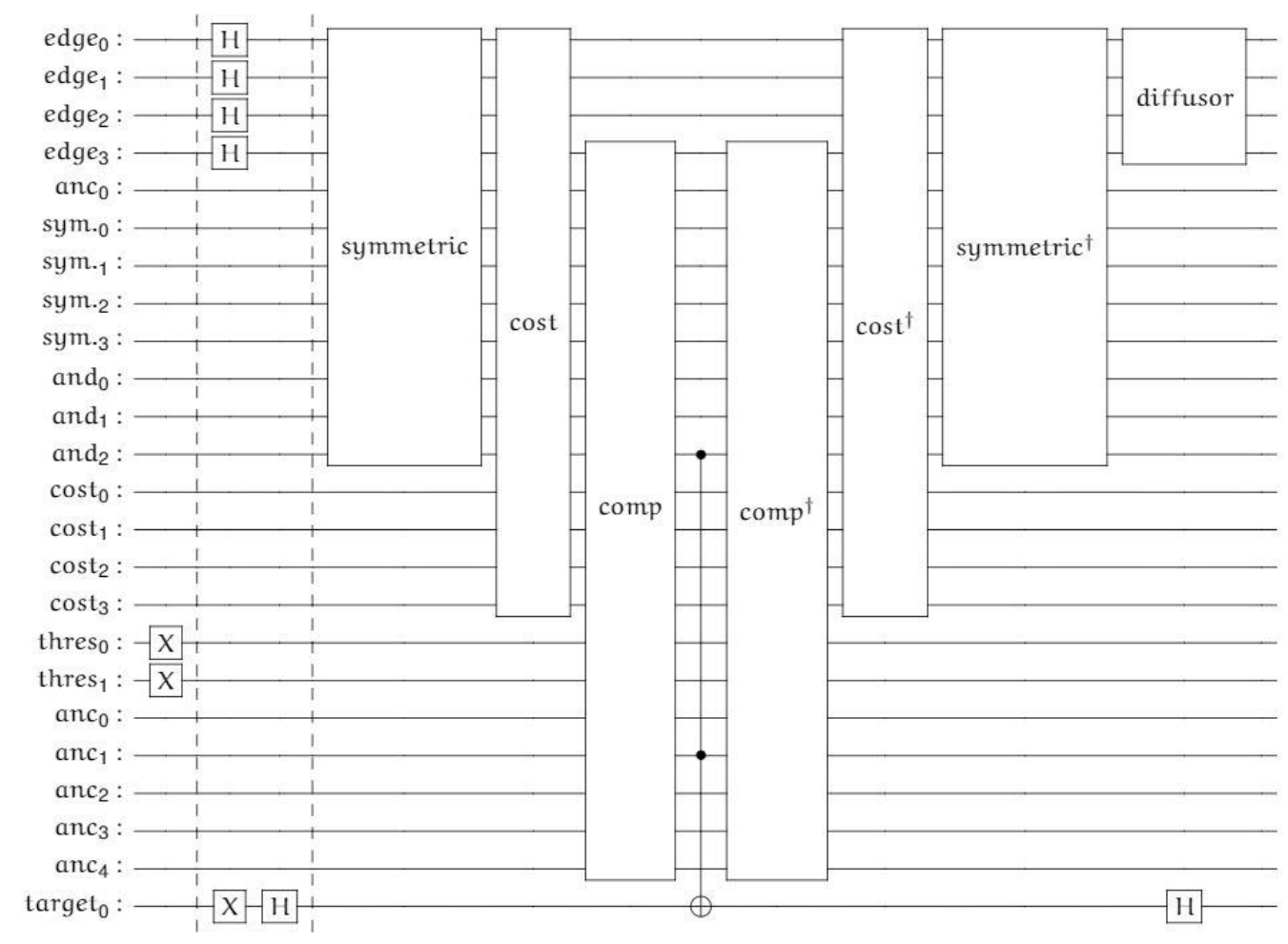

Fig. 6. The three components of the Grover oracle, symmetric, cost and comp have to be mirrored at the end of each iteration in order to restore the qubits state.

Research in quantum computing will continuously develop, and with the right technology such as the Noisy Intermediate-Scale Quantum (NISQ)-technology [17] quantum computers with limited computational power will be commercially available in the near future. As the name suggests, those NISQ-computers will be more prone to noisy environment, therefore, the circuits must be designed short in order to lower the impact of noise on the results. 
Two Quantum algorithms, Quantum Support Vector Machine algorithm [4] and the Swap Test method [13], were compared by solving the same problem. Both algorithms were designed and improved to classify 200 testing samples of two different numbers with only one training sample of each number. The result was a $100 \%$ accuracy for both algorithms but with less computational time, the Swap Test is better suited for decoherence prone quantum computers. Fig. [7] shows the circuit for the Quantum Support Vector Machine algorithm and Fig. [8] shows the circuit for the Swap-Test algorithm.

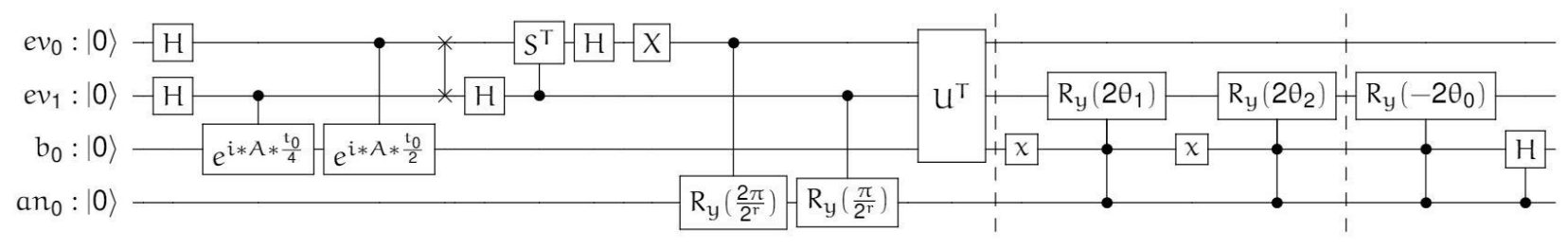

Fig. 7. A qubit in this Quantum Support Vector Machine circuit is $4.160 \mu \mathrm{s}$ exposed to noise.

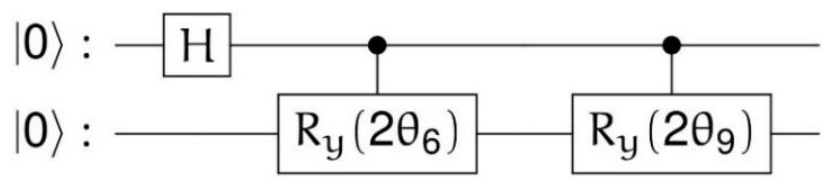

Fig. 8. A qubit in this Swap-Test circuit is $1.500 \mu$ s exposed to noise.

As the swap test is better suited for NISQ-computers, further research was conducted with this algorithm. To extend the problem of classifying two numbers, a methodology was designed in order to distinguish three different digits. The Swap Test method was able to classify those different digits of the OCR-dataset without a misclassification using an additional data mapping step. Fig. [9] illustrates the first step of this methodology in which the digits were divided into two groups according to the horizontal and vertical ratio of the pixels.

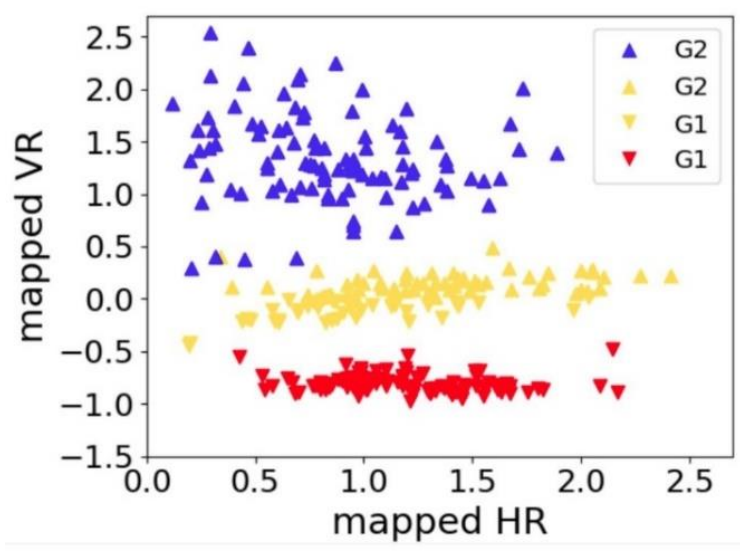

Fig. 9. The blue triangles represent the digits 8 the yellow triangles represent the digits 6 and the red triangles represent the digits 9. It is not possible to divide the digits with a straight line without any misclassification.

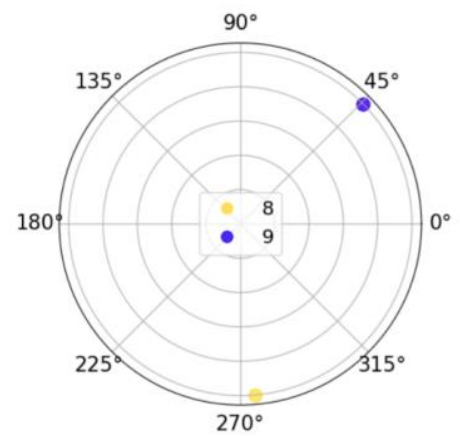

(a) Training data dirstribution

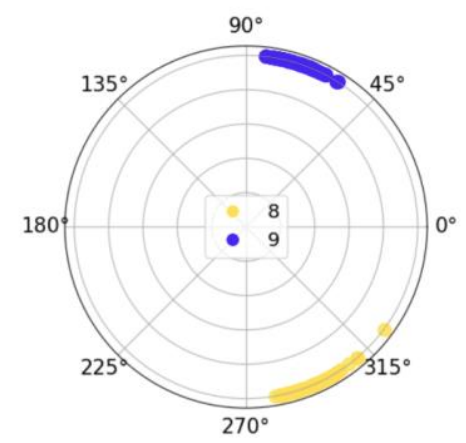

(b) Testing data dirstribution

Fig. 10. The blue triangles represent the digits 8 the yellow triangles represent the digits 6 and the red triangles represent the digits 9 . It is not possible to divide the digits with a straight line without any misclassification. 
After the digits were grouped a linear mapping was per formed to further divide the subgroups. Fig. [10] shows how the subgroup, containing the digits 8 and 9, was mapped onto the circumference of a qubit. To extend the Swap-Test methodology, a more complex dataset, the Breast Cancer dataset [19], was mapped onto the entire Bloch Sphere and not only the circumference. After conducting a Principal Component Analysis (PCA), the four highest components were used to transform these testing samples along the Bloch sphere. Fig. [11] shows the circuit that transforms the qubits state according to the four PCA-variables and also performs the Swap-Test.

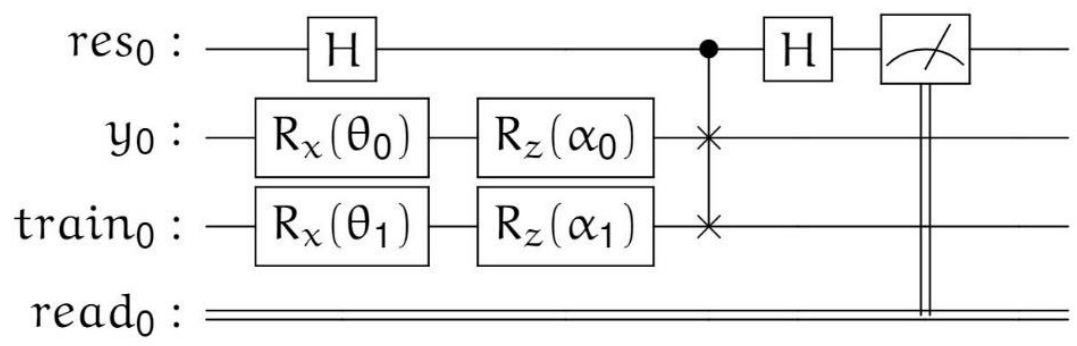

Fig. 11. The result of the Swap-Test is encoded on the qubit res ${ }_{0}$

The entire Breast Cancer dataset [19] was classified with this circuit with an accuracy of $92 \%$ which is only 5\% below the accuracy of the best-known classical algorithm. Fig. [12] shows the entire dataset being mapped onto a Bloch sphere.

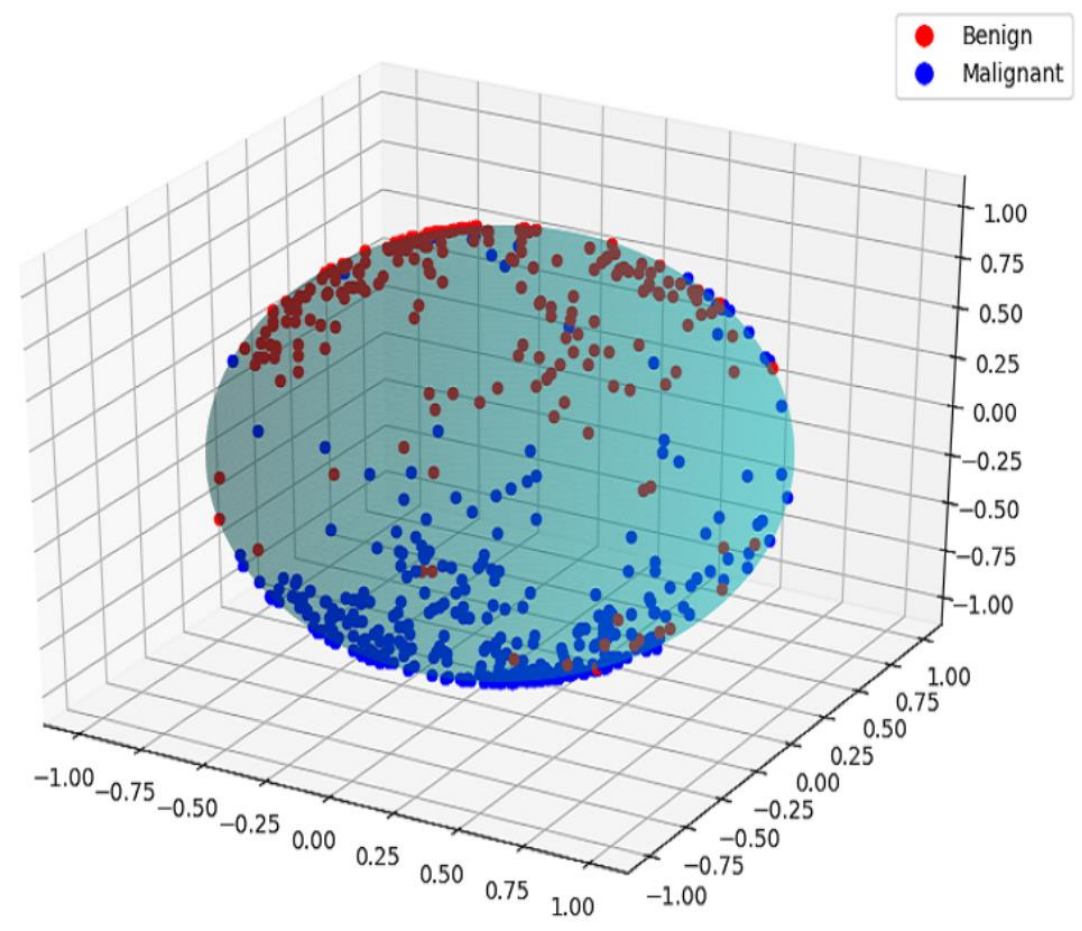

Fig. 12. All data points of the Breast Cancer Dataset mapped onto a sphere.

\section{Conclusion}

This research proposes potential solutions to two fields of modern robotics, namely Human-Robot-Interaction, and machine learning. More specifically, three questions were used to focus the research. For Human-Robot-Interaction we asked 1) how to control emotion and mood, while for machine learning we considered 2) how to solve the shortest path calculation and 3) how to apply a dataset to quantum computing or more precisely to a qubit. Within this research we presented two quantum circuits and showed how to set up quantum circuits on a real humanoid robot in order to express emotions. The hybrid-controlled robot was able to express certain behaviors like happiness and exhaustion. In future studies the aim will be to verify the theory of quantum cognition. This theory states that human behaviour has more parallels to quantum- than to classical-computing. In order to verify the theory of quantum cognition, more powerful quantum behavioural modelling circuits as well as quantum motion generation circuits will be required. Furthermore, it will be interesting to investigate the degree to which behaviour models should imitate humanlike behaviour. Is it enough that the humanoid robot executes motions according to left or right handedness or does the handedness also need to affect the thinking process of the robot as it is with human beings? 
One possibility could be to expand behavioural circuits according to the wheel of emotions of Plutchik [20]. Mapping the internal state according to the wheel of emotions of Plutchik likely enables better scalability in comparison to the mood-circuit presented in this paper. Another contribution of this paper is in the field of quantum machine learning. A Grover-Circuit was built in order to solve the shortest path problem. This Grover-circuit could not match the best classical algorithm in existence today, but it performed better than most of the classical algorithms for the shortest path problem. Additionally, different circuits were compared in order to figure out which algorithms are best suited for NISQ computers. Finally, we were able to classify the Breast-Cancer dataset using the swap test algorithm and thereby mapping the data points onto the entire Bloch-Sphere of a qubit. Here again it was not possible to show quantum supremacy, but this was not the goal of this paper. The primary objective was to bridge the gap between robotics and quantum using a humanoid robot and a real quantum computer. In future work the quantum library must be changed in order to achieve even better performance. The Qiskit simulation has problems with more than 30 qubits. In Bravyi \& Gosset [21] a new idea for simulating quantum circuits was introduced that can simulate even bigger circuits with up to a few hundreds of qubits. With more qubits a standalone Grover circuit can be built to solve the Shortest Path problem autonomously with all variations. Even with a simulator of better performance, the concept of the Shortest Path must be changed in order to get rid of the polynomial running time and be as efficient as the best classical algorithms.

The research question "How to implement quantum computing on a robot to create a behavioral model and solve algorithmic problems?" was answered with various circuits that ran on the humanoid robot. Larger circuits with more than 10 qubits were simulated while smaller ones were executed on real quantum computers. With several simulated and real quantum circuits this research not only verified a method for controlling a robot with the combination of a classical and a quantum computer, but also showed some practical robotic-relevant applications like solving the shortest path problem or classifying various datasets.

\section{References}

[1] Malham, S. (2015). An introduction to Lagrangian and Hamiltonian mechanics, 10.13140/RG.2.1.2914.8003.

[2] Karpova, I \& Karpov, V. (2018). Some mechanisms for managing aggressive behavior in group robotics, 10.2507/29th.daaam.proceedings.082.

[3] Mori, M.; MacDorman, K. \& Kageki, N. (2012). The Uncanny Valley [from the field], IEEE Robotics \& Automation Magazine, vol. 19, no. 2, pp. 98-100, 10.1109/MRA.2012.2192811.

[4] Li, Z.; Liu, X.; Xu, N. \& Du, J. (2015). Experimental realization of a quantum support vector machine, Physical review letters, 10.1103/physrevlett.114.140504.

[5] Rebentrost, P.; Mohseni, M. \& Lloyd, S. (2013). Quantum support vector machine for big data classification, Physical Review Letters. 113. 10.1103/PhysRevLett.113.130503.

[6] Bevacqua, E. \& Mancini, M. (2004). Speaking with emotions.

[7] Lukac, M. \& Perkowski, M. (2007). Quantum mechanical model of emotional robot behaviors, 37th International Symposium on Multiple Valued Logic (ISMVL 07), pp. 19-19, 10.1109/ISMVL.2007.44.

[8] http://community.qiskit.org/textbook, (2020). Learn quantum computation using Qiskit, Accessed on: 2020-04-15

[9] Braitenberg, V. (1986). Vehicles: Experiments in synthetic psychology, MIT Press, 9780262521123.

[10] Bruza, P.; Wang, Z. \& Busemeyer, J. (2015). Quantum cognition: a new theoretical approach to psychology, Trends in Cognitive Sciences, 10.1016/j.tics.2015.05.001

[11] Grover, L. (1996). A fast quantum mechanical algorithm for database search, STOC '96, Association for Computing Machinery, 10.1145/237814.237866.

[12] Shor, P. (1997). Polynomial-Time algorithms for prime factorization and discrete logarithms on a quantum computer, Society for Industrial \& Applied Mathematics (SIAM), 10.1137/s0097539795293172.

[13] Wittek, P. (2014). Quantum machine learning: What quantum computing means to data mining, Academic Press, 9780128009536.

[14] http://roboticsbusinessreview.com, (2018). Applying machine learning to robotics, Accessed on: 2020-06-03.

[15] Corey, D.; Hurley \& M.; Foundas, A. (2001). Right and left handedness defined: A multivariate approach using hand preference and hand performance measures, Neuropsychiatry, neuropsychology, and behavioral neurology, 14(3), 144-152.

[16] Aharonov, Y.; Davidovich, L. \& Zagury, N. (1993), Quantum random walks, Physical review, A. 48, 1687-1690. 10.1007/BFb0083545.

[17] Preskill, J. (2018). Quantum computing in the NISQ era and beyond, Quantum Physics, 10.22331/q-2018-08-06-79.

[18] Brassard, G.; Hoyer, P. \& Tapp, A. (1998). Quantum counting, Lecture Notes in Computer Science, Springer Berlin Heidelberg, 9783540686811.

[19] https://archive.ics.uci.edu/ml/datasets/Breast+Cancer+Wisconsin, (1995). Breast Cancer Wisconsin (Diagnostic) Data Set, University of Wisconsin, Accessed on: 2020-07-11.

[20] Plutchik, R. (1982). A psychoevolutionary theory of emotions. Social Science Information, 21(4-5), pp.529-553, $10.1177 / 053901882021004003$

[21] Bravyi, S. \& Gosser, D. (2016). Improved classical simulation of quantum circuits dominated by Clifford gates, Physical Review Letters, 116(25), 10.1103/PhysRevLett.116.250501. 